\title{
Measuring the differential cross section for top quark production at $8 \mathrm{TeV}$
}

\section{levgen KOROL*}

Deutsches Elektronen-Synchrotron Hamburg and Zeuthen (DE)

E-mail: ievgen.korol@cern.ch

\begin{abstract}
Normalized differential top quark pair production cross sections are measured in pp collisions at a centre-of-mass energy of $8 \mathrm{TeV}$ at the LHC using the CMS detector. The dataset used for these measurements corresponds to an integrated luminosity of $19.7 \mathrm{fb}^{-1}$. The measurements are performed in the lepton+jets ( $e+$ jets and $\mu+$ jets) and in the dilepton $(e e, \mu \mu$, and $e \mu)$ decay channels. The $t \bar{t}$ production cross section is measured as a function of kinematic properties of the charged leptons, the jets associated to $b$-quarks, the top-quarks, and the $t \bar{t}$ system. The data are compared with several predictions from perturbative QCD calculations up to approximate nextto-next-to-leading-order precision. No significant deviations with respect to the standard model predictions are observed.
\end{abstract}

8th International Workshop on Top Quark Physics, TOP2015

14-18 September, 2015

Ischia, Italy

${ }^{*}$ Speaker. 


\section{Introduction}

The Run-I data samples at the LHC contain a large amount of top-quark pairs. Analyzing these samples allows studies of top-quark production and properties at unprecedented precision. The data sample used for this analysis was recorded by the CMS detector at $8 \mathrm{TeV}$ center-of-mass energy. The luminosity of this sample is $19.7 \mathrm{fb}^{-1}$.

Understanding the production and properties of top quarks is fundamental for testing the quality of the Standard Model (SM). In particular, measurements of the top-quark pair $(t \bar{t})$ production cross section as a function of $t \bar{t}$ kinematic observables can be compared with quantum chromodynamic (QCD) predictions, providing tests and constraints for the theory parameters. Additionally, the differential measurements of the $t \bar{t}$ production are expected to be sensitive to the physics beyond SM [1].

In the following the differential $t \bar{t}$ production cross section in the dileptonic $(e e, \mu \mu$ and $e \mu)$ and the semileptonic ( $e$ or $\mu+$ jet) $t \bar{t}$ decay channels are presented.

\section{Event Selection and Reconstruction}

For the differential cross section measurements presented in this work events with semileptonic $(e / \mu+\mathrm{jet})$ and dileptonic $(e e, e \mu$ or $\mu \mu) t \bar{t}$ final states were selected [2].

For the dilepton final state the presence of at least two leptons (electrons or muons) with opposite charge and at least two anti- $k_{T}$ jets with radius parameter $\Delta R=0.5$ in the event is required. Also, at least one of the jets in the event should fulfill the loose CSV $b$-tagging criterion [3].

For the semileptonic final state the presence of exactly one electron or muon and at least four anti- $k_{T}$ jets with the radius parameter $\Delta R=0.5$ in the event is required. Additionally, at least two of the jets in the event have to be tagged as $b$-jets using the loose CSV criterion.

The following restrictions on the kinematics of the event were applied for the event selection:

- Leptons have to be isolated with a relative isolation $I_{r e l}(0.3)<0.15$ for the dileptonic and $I_{\text {rel }}(0.3)<0.10$ for the semileptonic final states.

- $p_{T}>20 \mathrm{GeV}$ and $|\eta|<2.4$ for all the leptons in the event for the dileptonic final state reconstruction and $p_{T}>30 \mathrm{GeV}$ and $|\eta|<2.1$ for all the leptons in the event for the semileptonic final state reconstruction.

- Dilepton mass veto: $m(l l)>20 \mathrm{GeV}$.

- All the jets in the event have to fulfill the requirements of $p_{T}>30 \mathrm{GeV}$ and $|\eta|<2.4$.

- For the dileptonic $t \bar{t}$ decay channel with $e e$ or $\mu \mu$ in final state, a veto on the $Z$-peak, $\mid m_{Z}-$ $m(l l) \mid>15 \mathrm{GeV}$, and the transverse momentum restriction $E_{T}^{\text {miss }}>40 \mathrm{GeV}$ are applied.

After selecting the events, which fulfill the requirements described above, the full kinematics of the $t \bar{t}$ pair (i.e. of the $t$ and the $\bar{t}$ ) is reconstructed using the kinematics of jets and leptons and missing transverse energy.

In the semileptonic $t \bar{t}$ decay channel, a constrained fit is applied [4] to the four-momenta of the selected lepton, up to five leading $p_{T}$ jets and missing transverse momentum, which is assumed 
to occur from the neutrino from the leptonic top decay. In the fit, the mass of the the $W$ boson is constrained to $80.4 \mathrm{GeV}$ and the masses of the top quarks are required to be equal. Only $b$-tagged jets are considered as $b$-quark candidates. If the event allows several combinatorial solutions, only the one with the smallest $\chi^{2}$ of the fit is accepted.

The dileptonic $t \bar{t}$ final state has two undetected neutrinos, which can be represented by the total missing transverse momentum. Thus, this system is underconstrained and additional assumptions are needed to reconstruct the full kinematics of the $t \bar{t}$. Six kinematic constraints are applied: the $W^{ \pm}$mass constraint, the top- and antitop-quark mass constraints and the constraint on the sums of the $x$ and $y$ momenta components of neutrino and antineutrino to be equal to the missing transverse momenta components in the event. These constraints result in a system of equations which can be solved algebraically [5]. The details of the kinematic reconstruction algorithm applied are described in [2].

\section{Cross Section Determination}

The normalized differential $t \bar{t}$ production cross section the $i^{t h}$ bins of the variable $X$ is determined as follows:

$$
\left(\frac{1}{\sigma} \frac{d \sigma}{d X}\right)_{i}=\frac{1}{\sigma} \cdot \frac{1}{\Delta X_{i}} \cdot \frac{N^{d a t a}}{L} .
$$

Here $N^{\text {data }}$ is the number of selected and reconstructed events in data after background subtraction and corrected for detector efficiencies, acceptances and migrations, $\Delta X$ is the bin width of the bin $i$ of the variable $X$ and $L$ is the integrated luminosity. A normalization to the in-situ measured inclusive $t \bar{t}$ production cross section $\sigma$ is performed. To correct for the migrations due to detector effects, a regularized unfolding based on singular value decomposition [6] is utilized.

\section{Results}

The normalized differential $t \bar{t}$ production cross sections in bins of different variables describing the kinematics of the top-quark and the $t \bar{t}$ system are presented in Fig. 1 .

While the top $p_{T}$ spectrum is observed to be better described with the Powheg+Herwig6 setup, the Madgraph+Pythia6 is generally in better agreement with the observed $m(t \bar{t})$ spectrum. The $p_{T}$ spectra of top quarks in data are softer than in the model predictions. The best description of the $p_{T}$ spectra from the data are provided with the NNLO calculations. The $m(t \bar{t})$ in data tends to be lower than the predictions for large $m(t \bar{t})$. More cross sections were measured and discussed in [2].

\section{References}

[1] R. Frederix and F. Maltoni, Top pair invariant mass distribution: a window on new physics, JHEP 01 (2009) 047, doi:10.1088/1126-6708/2009/01/047, arXiv:0712.2355

[2] V. Khachatryan and others, Measurement of the differential cross section for top quark pair production in pp collisions at $\sqrt{s}=8 \mathrm{TeV}$, Eur. Phys. J. C75 (2015) 11, doi:10.1140/epjc/s10052-015-3709-x, arXiv:1505.04480 

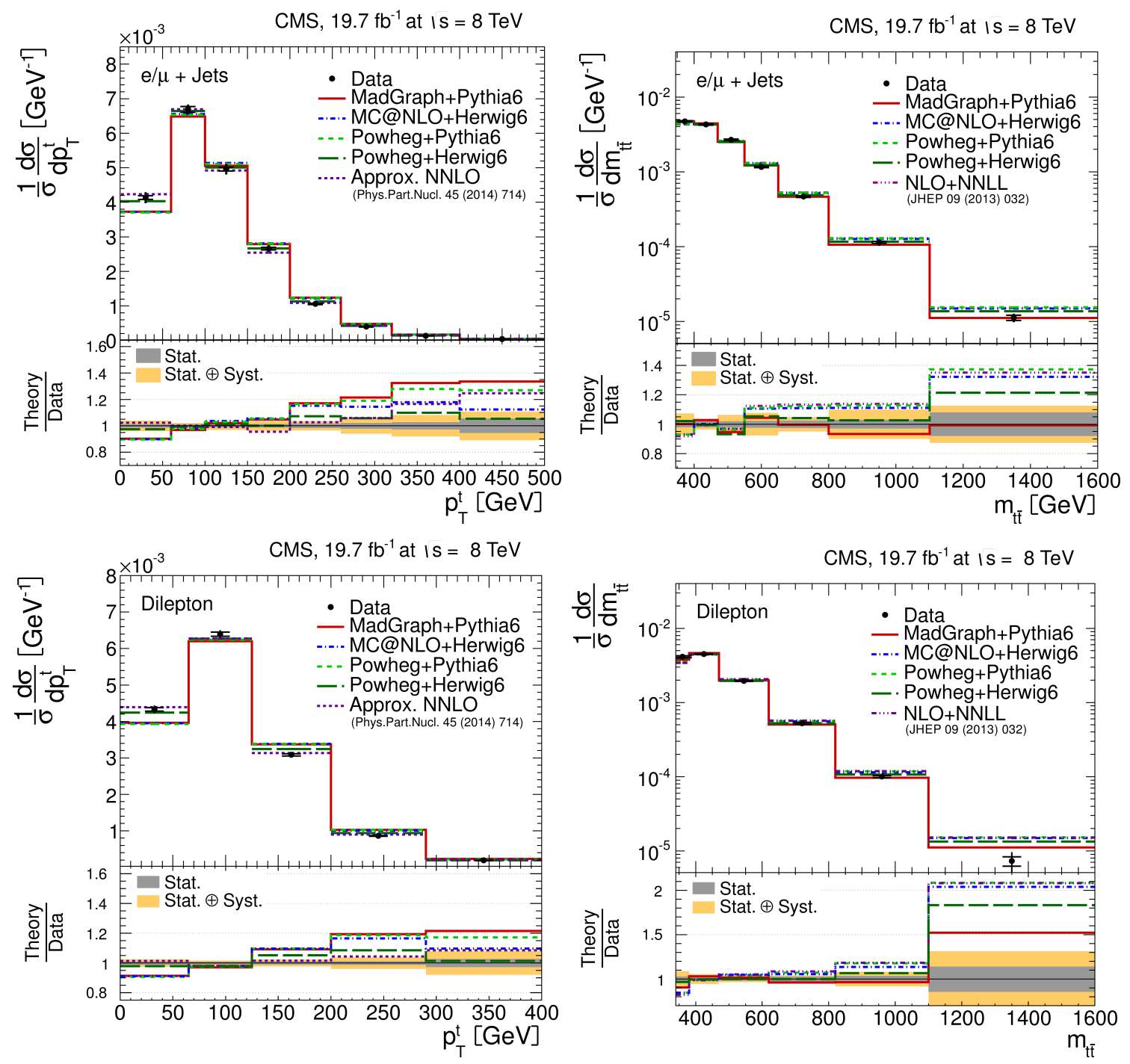

Figure 1: Normalized differential cross sections in bins of top-quark transverse momentum reconstructed in the semileptonic (top left) and dileptonic (bottom left) decay channels and invariant mass of the $t \bar{t}$ system reconstructed in the semileptonic (top right) and dileptonic (bottom right) decay channels. The data points are placed at the midpoint of the bins. The inner (outer) error bars indicate the statistical (combined statistical and systematic) uncertainties. The measurements are compared to different MC setups or fixed order calculations, when available. The bottom panel of each plot shows the ratio of the predictions to data.

[3] S. Chatrchyan and others, Identification of b-quark jets with the CMS experiment, JINST 8 (2013) P04013, doi:10.1088/1748-0221/8/04/P04013, arXiv:1211.4462

[4] J. D'Hondt and others, Fitting of event topologies with external kinematic constraints in CMS, CERN-CMS-NOTE-2006-023 (2006)

[5] L. Sonnenschein, Algebraic approach to solve t $\bar{t}$ dilepton equations, Phys. Rev. D72 (2005) 095020 , doi:10.1103/PhysRevD. 72.095020, arXiv:hep-ph/0510100

[6] A. Hocker and V. Kartvelishvili, SVD approach to data unfolding, Nucl. Instrum. Meth. A372 (1996) 469-481, doi:10.1016/0168-9002 (95) 01478-0, arXiv:hep-ph/9509307 
[7] A. Ferroglia, B.D. Pecjak and L.L. Yang, Top-quark pair production at high invariant mass: an NNLO soft plus virtual approximation, JHEP 09 (2013) 032, doi:10.1007/JHEP09 (2013) 032, arXiv:1306.1537

[8] N. Kidonakis, NNLL threshold resummation for top-pair and single-top production, Phys. Part. Nucl. 45 (2014) 4 714-722, doi : 10 .1134 / S1 063779614040091 , arXiv:1210.7813 